\title{
PAPER \\ TongSACOM: A TongYiCiCiLin and Sequence Alignment-Based Ontology Mapping Model for Chinese Linked Open Data
}

\author{
Ting $\mathrm{WANG}^{\dagger \mathrm{a})}$, Tiansheng $\mathrm{XU}^{\dagger \mathrm{b})}$, Nonmembers, Zheng TANG ${ }^{\dagger \dagger \mathrm{c}}$, Member, , and Yuki TODO $^{\dagger \dagger \mathrm{d})}$, Nonmember
}

\begin{abstract}
SUMMARY Linked Open Data (LOD) at Schema-Level and knowledge described in Chinese is an important part of the LOD project. Previous work generally ignored the rules of word-order sensitivity and polysemy in Chinese or could not deal with the out-of-vocabulary (OOV) mapping task. There is still no efficient system for large-scale Chinese ontology mapping. In order to solve the problem, this study proposes a novel Tong YiCiCiLin (TYCCL) and Sequence Alignment-based Chinese Ontology Mapping model, which is called TongSACOM, to evaluate Chinese concept similarity in LOD environment. Firstly, an improved TYCCLbased similarity algorithm is proposed to compute the similarity between atomic Chinese concepts that have been included in TYCCL. Secondly, a global sequence-alignment and improved TYCCL-based combined algorithm is proposed to evaluate the similarity between Chinese OOV. Finally, comparing the TongSACOM to other typical similarity computing algorithms, and the results prove that it has higher overall performance and usability. This study may have important practical significance for promoting Chinese knowledge sharing, reusing, interoperation and it can be widely applied in the related area of Chinese information processing.

key words: Semantic Web, linked open data, ontology mapping, similarity computing, TongYiCiCiLin
\end{abstract}

\section{Introduction}

The vision of the Semantic Web is to establish a web of data that would allow machines to understand semantic information on the Internet [1]. Ontology, as the core factor of the Semantic Web, is considered as a formalization and normalization method that describes the shared concepts of specific domains [2]. It is the foundation for realizing knowledge sharing and semantic interoperation. Currently, research on linked open data (LOD) [3] is primarily centered on the levels of instances [4], [5], but studies on linked data at the schema level are equally important [6]. As a typical scenario of linking open data on schema-level, ontology alignment has been widely researched.

Melnik et al. [7] proposed a structural level ontology mapping algorithm called "similarity flooding" that uses the

Manuscript received July 15, 2016.

Manuscript revised February 2, 2017.

Manuscript publicized March 15, 2017.

${ }^{\dagger}$ The authors are with the Information School, Capital University of Economics and Business, Beijing, 100070 China.

${ }^{\dagger \dagger}$ The author is with the Faculty of Engineering, University of Toyama, Toyama-shi, 930-8555 Japan.

${ }^{\dagger \dagger}$ The author is with the Faculty of Electrical and Computer Engineering, Kanazawa University, Kanazawa-shi, 920-1192 Japan.

a) E-mail: wangting@ cueb.edu.cn

b)E-mail: xuts@cueb.edu.cn

c) E-mail: zhengtang2000@ hotmail.com

d) E-mail: yktodo@ec.t.kanazawa-u.ac.jp (Corresponding author)

DOI: 10.1587/transinf.2016EDP7307 concept of ontology to build a similarity propagation map and then spreads and corrects any similarities between the concepts. Cohen et al. [8] listed some typical similarity computing algorithms on the element-level based on EditDistance and Token, and then evaluated their performance. Giunchiglia et al. [9] proposed that semantic relationships should be discovered based on linguistic method by introducing shared knowledge dictionary (e.g., WordNet [10]). Isaac et al. [11] proposed an Instance-Level ontology mapping algorithm that would measure the similarities between the concepts according to the number of similar instance of ontology concepts. Based on the Workflow technique, Nikolov et al. [12] proposed a linked data framework: KnoFuss, which make use of the hierarchy between concepts in order to pick up the most suitable mapping parameter and approach. Zhong et al. [13] developed the RiMOM system, which is a multi-strategy mapping system based on ontology instance, concept name, ontology structure and other characteristics, and applied it for large-scale ontology mapping by introducing universal field theory. Based on bootstrapping and taking Wikipedia classification tree as the knowledge base for semantic similarity computing, Jain et al. proposed the BLOOMS system [6], which can efficiently build the Schema-Level interlinking, that is ontology mapping, in LOD environment. But all the researches mentioned above are limited to the schema matching between datasets described in the English language only.

However, due to the cultural background, a large-scale ontology alignment system for Chinese language has not yet to be developed. In recent years, more researches have been conducted in association with large-scale Chinese ontology and linked data. Li et al. [14] proposed a similarity computing method on the element-level based on HowNet [15] and established a Chinese ontology mapping system, which is called ELOMC; unfortunately, it ignored the rules of wordorder sensitive and polysemy in Chinese language proposed by Chinese linguists $\mathrm{Lu}$ [16]; therefore, its practicability and effectiveness still need to be verified while facing the largescale ontology mapping task. Based on TongYiCiCiLin (TYCCL), a Chinese synonym thesaurus, Tian et al. [17] proposed an algorithm for computing the similarity between atomic Chinese concepts, but whose method do not apply to the LOD environment and could not deal with the out-ofvocabulary (OOV).

In addition, there are also some typical systems, which should be mentioned, establishing the interlinking between LOD datasets mainly at the instance level. Silk [18], [19] 
is a framework for building the interlinking between different datasets, by a declarative language, users can configure the linking strategy, such as the type, conditions etc., and it also supports the remote interlinking. Hassanzadeh et al. [20] proposed a common and extensible system: LinQL, which has integrated some existed link discovery method. The purpose of this system is for helping users to select the most suitable method to interlink their own dataset. Meanwhile, it also support publishing RDF triples from Relational Data Base (RDB) by using D2RQ [21] and Virtuoso. In order to achieve the sharing, reusing, and interoperation of knowledge bases in LOD environment, it is necessary to link ontologies described in different languages. Wang et al. [22] proposed a method for cross-linguistic interlinking establishing in LOD. Firstly, by using the concept annotation method, with the help of a small amount of cross-language and internal links seed to enrich the internal links, secondly, a regression model is used to predict the potential crosslanguage links between Chinese and English Wiki. Wang et al. [5] proposed extracting the hierarchical relationships between concepts and the concept property contained in Infobox based on the DMOZs (a kind of open classification project) of Chinese Wiki; furthermore, based on a simple keyword-matching method, eventually building a BaiduBaike and Hudong-based ontology and establishing the co-reference relationships between instances with DBpedia [23]. Niu et al. [4] conducted semantic integrations of Baidu Baike ${ }^{\dagger}$, Hudong ${ }^{\dagger \dagger}$, and DBpedia ${ }^{\dagger \dagger \dagger}$, and developed a Chinese linked data application system, Zhishi.me, at the instance-level. But all the researches mentioned above are limited to the instance-level matching between datasets and establishing of interlinking at schema-level of them is still insufficient.

Unless the ontological knowledge described in Chinese is addressed, it will be impossible to collect semantic data described by Chinese ontology. Therefore, mapping Chinese ontology as well as the ontology described with other languages is integral to sharing and interoperation among ontologies. Moreover, the researches on Chinese linked data on the web are still in the early stage; the approaches for linked the Schema-Level data in Chinese are very insufficient.

Based on the above, this study proposes a novel linked open data model (TongSACOM) for large-scale Chinese ontology mapping in LOD environment and experiments have shown that TongSACOM has more advantages and rationality than those traditional methods while facing the Chinese ontology schema matching task.

\section{Definition of the Problem}

In Chinese ontology mapping, simple lexical units and

\footnotetext{
${ }^{\dagger}$ See http://baike.baidu.com/

${ }^{\dagger}$ See http://www.hudong.com/

${ }^{\dagger \dagger}$ See http://wiki.dbpedia.org/
}

OOV words correspond to the ontology concepts to be mapped. Therefore, the simple lexical unit is termed as the atomic concept $(A C)$ and the concept that corresponding to the OOV word is termed as the compound concept $(C C)$. TongSACOM defines that all $C C_{s}$ comprise a linear array and a combination of multiple atomic concepts [14]. Firstly, the formal descriptions of the problems faced are given.

Definition 1: Ontology mapping: There are two ontologies for mapping, namely, $O_{s}$ and $O_{t}$. For each concept $C_{s}$ in $O_{s}$, finding a corresponding concept $C_{t}$ in $O_{t}$ that has the same or similar semantic meaning as it. Define the mapping function map: $O_{s} \rightarrow O_{t}$ :

For $C_{s} \in O_{s}, C_{t} \in O_{t}$, if $\operatorname{sim}\left(C_{s}, C_{t}\right)>t ; \operatorname{map}\left(C_{s}\right)=C_{t}$

$t$ is a threshold value indicates that, when the semantic similarity between $C_{s}$ and $C_{t}$ is greater than $t,\left\langle C_{s}, C_{t}\right\rangle$ will be considered as the discovered concept mapping pair.

Definition 2: For the TYCCL semantic knowledge base (SKB), the set $S K B_{T Y C C L}$ comprises the atomic concepts. In other words, $S K B_{T Y C C L}=\left\{A C_{1}, A C_{2}, \ldots, A C_{N}\right\}$, in which an element $A C_{i}$ is an atomic concept in the set $S K B_{T Y C C L}$ and $N$ is the number of lexical units contained in the knowledge base.

Definition 3: The compound concept $C C_{i}$ comprises an ordered combination of atomic concepts. In other words, for $\forall A C_{i} \in S K B_{T Y C C L}$, introduce two-dimensional indices $i$ and $j$, and then there is an ordered sequence $C C_{i}=$ $\left[A C_{i 1}, A C_{i 2}, \ldots, A C_{i j}\right]$, where $j \geq 1$ and $C C_{i} \notin S K B_{T Y C C L}$; $j$ is the position of atomic concept $A C_{i}$ in the ordered sequence $C C_{i}$; in particular, for all atomic concepts $A C_{i}$, $A C_{i}=\left[A C_{i}\right]$

Definition 4: As with $C_{s}$ and $C_{t}$ in $O_{s}$ and $O_{t}$, the ontology concepts for mapping are $C_{s}=C C_{s}=\left[A C_{s 1}, A C_{s 2}, \ldots\right.$, $\left.A C_{s m}\right]$ and $C_{t}=C C_{t}=\left[A C_{t 1}, A C_{t 2}, \ldots, A C_{t n}\right]$, where $m$ and $n$ are the length of the ordered sequences $C C_{s}$ and $C C_{t}$, which correspond to $C_{s}$ and $C_{t}$, respectively, and then $m$, $n \geq 1$.

\section{A TongYiCiCiLin and Sequence Alignment-Based Chinese Ontology Mapping Model (TongSACOM)}

On the basis of formal descriptions and definitions of the problems given above, the TongSACOM model is proposed and each situation of the problem will be discussed. The architecture of the model is shown in Fig. 1.

The TongSACOM model is mainly consisted of the following modules: Ontology Pre-Processing, Improved TYCCL Similarity Computing, Splitting Compound Concept, Building Scoring Matrix, Compound Concept Similarity, and Sequence Alignment Processing.

While mapping any two concepts $C_{s}$ and $C_{t}$ in $O_{s}$ and $O_{t}$ will result in one of the following three cases:

(1) $C_{s}$ and $C_{t}$ are both the atomic concepts, i.e., $C_{s} \in$ $S K B_{T Y C C L}$ and $C_{t} \in S K B_{T Y C C L}$.

(2) One of the two concepts $\left(C_{s}\right.$ or $\left.C_{t}\right)$ is an atomic concept, but another one is a compound concept, i.e., $C_{s} \notin$ $S K B_{T Y C C L}$ xor $C_{t} \notin S K B_{T Y C C L}$.

(3) $C_{s}$ and $C_{t}$ are both compound concepts, i.e., $C_{s} \notin$ 


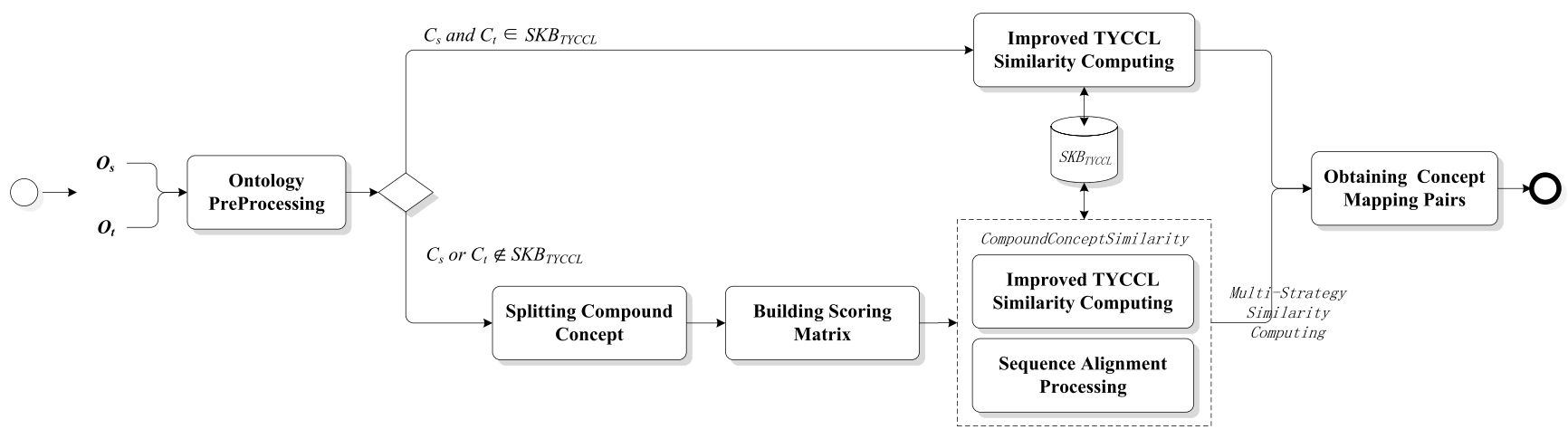

Fig. 1 The architecture of TongSACOM.

Table 1 An example of a TYCCL code

\begin{tabular}{c|c|c|c|c|c|c|c|c}
\hline Code Bit & 1 & 2 & 3 & 4 & 5 & 6 & 7 & 8 \\
\hline Sub-Code & B & a & 0 & 1 & A & 0 & 2 & $=$ or \# or @ \\
\hline Meaning & $\begin{array}{c}\text { Broad } \\
\text { heading }\end{array}$ & $\begin{array}{c}\text { Middle } \\
\text { heading }\end{array}$ & $\begin{array}{c}\text { Small } \\
\text { heading }\end{array}$ & $\begin{array}{c}\text { Word } \\
\text { group }\end{array}$ & $\begin{array}{c}\text { Atomic } \\
\text { word group }\end{array}$ & $\begin{array}{c}\text { synonym/unequal } \\
\text { /isolated }\end{array}$ \\
\hline Level & Level 1 & Level 2 & Level 3 & Level 4 & Level 5 & \\
\hline
\end{tabular}

$S K B_{T Y C C L}$ and $C_{t} \notin S K B_{T Y C C L}$.

For Case (1), it is implemented by the "Improved TYCCL Similarity Computing" module as shown in Fig. 1, and the improved TYCCL-based similarity algorithm is proposed to evaluate the semantic similarity between the two atomic concepts; the specific discussion about it is in the following Sect. 3.1. Furthermore, the solutions for Cases (2) and (3) are implemented by the "Compound Concept Similarity" module in which a sequence alignment and improved TYCCL-based combined algorithm is proposed to evaluate the similarity between compound concepts, the input of this module is the two unmapped sequences corresponding to the compound concepts and the scoring matrix, both of them are generated by the previous "Splitting Compound Concept" and "Building Scoring Matrix" modules respectively. The detail of this module is in the following Sect. 3.2.

\subsection{The Improved TYCCL Similarity Computing}

TYCCL is a Chinese synonym thesaurus in which each word is encoded and organized in a hierarchical tree and each node (lexical unit) represents a concept [24]. In TongSACOM model, an extended version of TYCCL that was published by $\mathrm{HIT}^{\dagger}$ will be used as a common semantic knowledge base $\left(S K B_{T Y C C L}\right)$ to acquire the equivalent relationships between concepts.

Improved TYCCL Similarity Computing: In experiment process, two concepts in different hierarchy of TYCCL are represented as <subclass of $>$ relationship, and the purpose of ontology mapping is to find the equivalent relationship between concepts but not the <subclass of $>$ relationship. While Tian et al. emphasizes too much on the semantic correlation between concepts [17], which will cause greater errors that a lot of < subclass of $>$ relationship will appear in result but not the equivalent relationship of

\footnotetext{
${ }^{\dagger}$ http://ir.hit.edu.cn/demo/ltp/Sharing_Plan.htm
}

concepts while carrying out the ontology mapping task. Therefore, an improved TYCCL algorithm is proposed by taking into account the influence of hierarchy in TYCCL on concept similarity and involving the semantic correlation factor $\lambda$. Finally through the concept similarity weight coefficient: $\lambda \times\left(L_{i} /|L|\right)$ to quantify it so that it can be applied to the ontology mapping task in LOD environment.

Based on the structural characteristics of TYCCL, the TYCCL concept codes for mapping are analyzed first. Extract the sub-codes of Level 1 to Level 5 and then start comparing with the code from the first sublevel. If one sub-code is different, the similarity weight of the mapping pair is determined by the sub-code's level. The deeper the sub-code's level, the higher the similarity weight, and vice versa. Meanwhile, the number of branch nodes of each layer also affects the similarity.

Taking the lexical unit “物质 (material)" as an example (TYCCL code: Ba01A02=), its TYCCL code format as indicated in Table 1 . The improved TYCCL-based similarity is computed by Eq. (1) as follows:

$$
\operatorname{SIM}_{T}\left(C_{s}, C_{t}\right)=\lambda \times \frac{L_{i}}{|L|} \times \cos \left(N_{T} \times \frac{\pi}{180}\right) \times\left(\frac{N_{T}-D+1}{N_{T}}\right)
$$

Because ontology mapping is more concerned with the semantic similarity between concepts, the adjustable parameters will be introduced into the model, specifically, semantic correlation factor $\lambda$, which is used to adjust the semantic relevance and similarity relationships between the different levels of concepts and to control the degree of similarity between lexical units at different branch levels. Clearly, $\lambda \in(0,1)$. The greater value of $\lambda$, the greater possibility similar or equivalent of lexical units on different levels will be. The influence of the semantic correlation on the final concept similarity will be greater and vice versa. However, the value of $\lambda$ in TongSACOM should not be set very high. The 
reasons are: (i) the "subclass-of" relationship between every two atomic concepts in different levels of the TYCCL will seriously interfere with accumulation of the "equivalentClass" relationship between every two atomic concepts in the Chinese ontology mapping process; and (ii) in the TongSACOM model, we set the threshold value $(t)$ of the "equivalentClass" relationship judgment between every two concepts to $t>=0.9$; therefore, if the $\lambda$-value is taken too high, it will increase the similarity value of all the mapping results, resulting in a large number of error mapping results output by SIM $_{T}$ algorithm (because the similarity of some error mapping results will be greater than 0.9 and result in the "subclass-of" relationship between every two atomic concepts in different levels of the TYCCL will be wrongly identified as the "equivalentClass" relationship) and finally reduce the performance of TongSACOM. Therefore, through contrasting several preliminary experiments, an essential conclusion is drew that: While the $\lambda$ is set to be a random number of $\lambda \in[0.4,0.5]$, TongSACOM can achieve the best overall performance.

$L=\{1,2,3,4,5\}$. For $\forall L_{i} \in L, L_{i}$ represents the level number of Level $i$. $|L|$ represents the number of elements in the set $L$. In this system, $|L|$ is constant and equal to 5 . The concept similarity weight coefficient is $\lambda \times\left(L_{i} /|L|\right) . N_{T}$ is the total number of nodes of the different sub-codes that appear in Level branch $i$ between $C_{s}$ and $C_{t}$. $D$ is the code distance between $C_{s}$ and $C_{t}$. In particular, when the codes of the five levels correspond to the concepts to be mapped and the last code bit of the TYCCL code is " $=$ ", the returned value of the similarity function $\operatorname{SIM}_{T}$ is 1.0. Clearly, the range of the function $\operatorname{SIM}_{T}$ is $(0,1]$.

\subsection{Sequence Alignment-Based Mapping for Chinese Compound Concept Similarity Computing}

Regarding the similarity in computing Chinese compound concepts, scholars have proposed their own solutions. For example, Li et al. proposed a Hownet-based concept similarity computing method on element-level and implemented a Chinese ontology mapping system (ELOMC) [14]. In particular, when they calculated the similarity between OOV, two compound concepts $A$ and $B$ to be mapped are expressed in the form of matrix; by definition 3 and the Chinese Segmentation Tool, they are both split into a group of atomic concepts and consist of two sequences respectively. The matrix is composed of the two sequences of compound concept $A$ and $B$; furthermore, Sequences $A$ and $B$ consist of the row and column of this similarity matrix respectively. Finding the relative highest similarity value of atomic mapping pair by traversing between the two sequences in matrix and then it could ultimately obtain the similarity values for the two compound concepts. The algorithm is as follow:

$$
\operatorname{Sim}(A, B)=\frac{\sum_{i=1}^{\max (m, n)} \max _{i}\left(M_{x y}\right)}{\max (m, n)}
$$

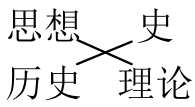

Fig. 2 The wrong mapping result.

where $M_{x y}$ represents a similarity value of a pair of mapping atomic concepts in the $m \times n$ matrix. $m$ and $n$ is the length of sequence $A$ and $B$ respectively. The $\max _{i}\left(M_{x y}\right)$ represents the ranked $i$-th similarity value in the matrix, and then computes Top $\max (m, n)$ similarity value. Some similar approaches have also been used in Refs. [25]-[27].

However, important information is often arranged in the second half rather than the first half of a sentence in Chinese [16], [28]; thus, this approach above will inevitably lead to semantic similarity computing errors because it ignored the rules of word-order sensitivity and polysemy in Chinese. For example, there are two compound ontology concepts to be mapped in Hudong-DBpedia sub-mapping task: history, namely, “历史理论 (history theory)” and “思 想史 (history of thought).” After they are split, two ordered atomic concept sequences are obtained: [历史, 理论] ([history, theory]) and [思想, 史] ([thought, history]). Based on the OOV word processing method that was presented in Eq. (2), the mapping result is shown in Fig. 2. Computing the semantic similarity of each pair of atomic concepts to be mapped based on Eq. (1), and then comprehensively compute the similarity using Eq. (2), given in Refs. [14]. The resulting similarity value of the concept is 1.0. It appears that the compound concept mappings and similarity results are not reasonable at all, which primarily because this approach ignores a word-order sensitivity and polysemy that is prevalent in Chinese mentioned before.

Thus, a novel method for improving the similarity computing between compound concepts is proposed. Specifically, while calculating the element-level similarity between the concepts, semantic similarity is computed by introducing a bioinformatics-based global dual-sequence alignment algorithm.

\subsubsection{Overview of the Sequence Alignment Algorithm}

In bioinformatics, dual-sequence alignment refers to two DNA or protein sequences that are arranged together to indicate their similarities; a space characters (-) can be inserted to have the same or similar symbols arranged in the same sequences. By comparing the similar fragments and conserved sites of two sequences, we can search for possible molecular evolutionary relationships [29].

\subsubsection{Building a Dynamic Programming Scoring Matrix}

A sequence refers to a string consisting of a series of words based on certain rules of arrangement.

Splitting the Compound Concept: Specifically, when computing similarity in ontology concepts, TongSACOM model considers the compound concept to be a sequence 


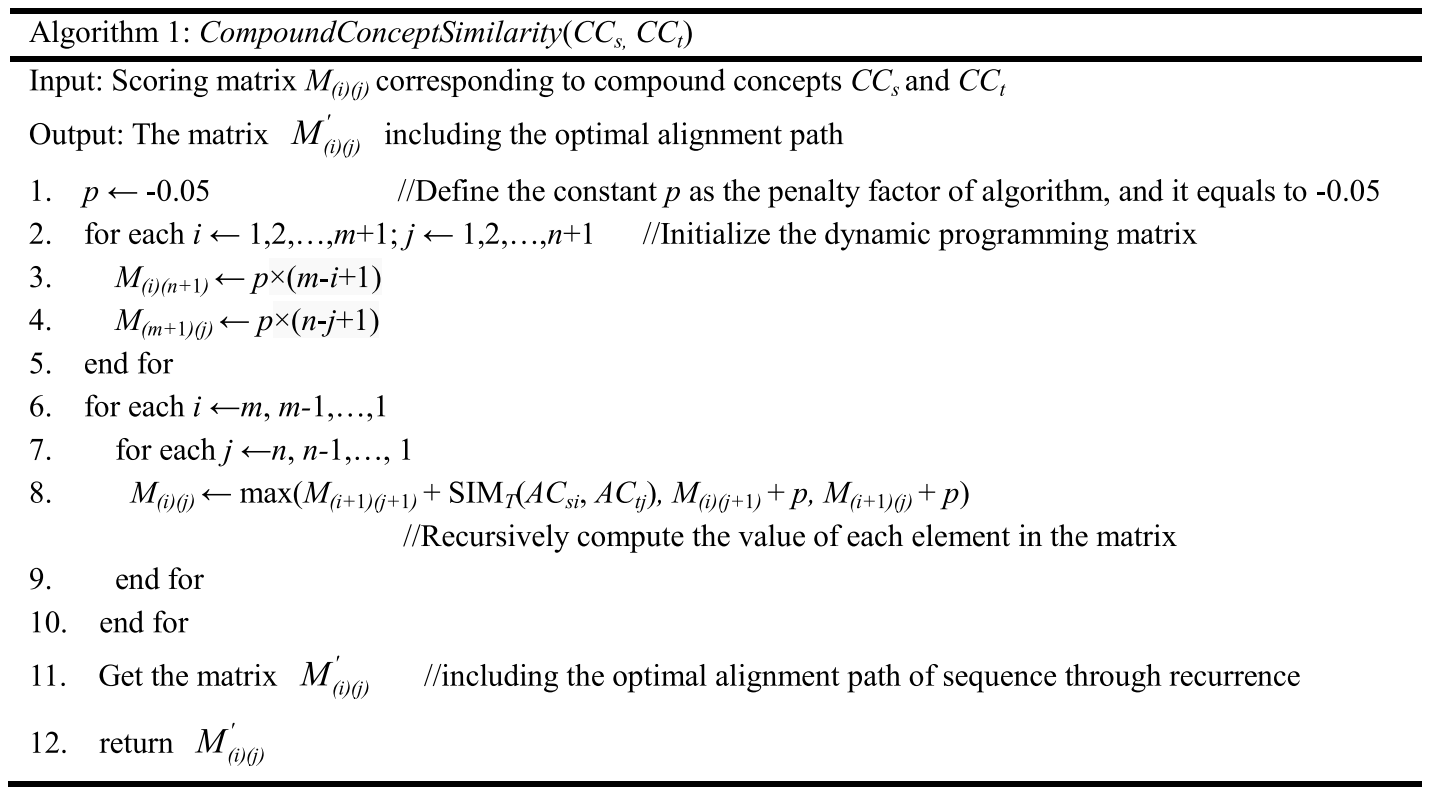

Fig. 3 The processing of getting the optimal alignment path between compound concepts.

of word strings in which each element is an atomic concept. First, splitting a compound concept into a list of simple lexical units and obtain its word string sequence which is generated by the "Splitting Compound Concept" module as shown in Fig. 1. In large-scale Chinese ontology mapping, the ICTCLAS50 software ${ }^{\dagger}$, developed by the Institute of Computing, Chinese Academy of Sciences, is used as the word segmentation tool. The alphabet is defined as the "Semantic Knowledge Base" of TYCCL ( $\left.S K B_{T Y C C L}\right)$.

TongSACOM abstracts the computation of similarity between ontology concepts into a process of aligning two sequences. The gap penalty function is used to determine the location for inserting space characters in the sequences to give them the same length, and a mapping relationship will be built between two atomic concepts or between an atomic concept and a space character in the aligning sequences. The sequence alignment algorithm essentially aims to find the global optimization alignment result between two sequences by using a scoring strategy.

Building the Scoring Matrix: In TongSACOM model, the two sequences to be aligned are expressed in the form of a scoring matrix firstly and then are treated as the two dimensions in the dynamic programming matrix: $M$.

For $C_{s}$ and $C_{t}$ in $O_{s}$ and $O_{t}$, Row $i$ in the scoring matrix $M$ corresponds to the atomic concept $A C_{s i}$ in the sequence $C C_{s}$. Column $j$ corresponds to the atomic concept $A C_{t j}$ in the sequence $C C_{t}$, where $i \leq m$ and $j \leq n$. The element in Row $i$ and Column $j$ of the dynamic programming matrix $M$ is called $M_{i j}$. Following the principles of dynamic programming, the model expresses the two sequences in rows and columns.

Assuming that sequence $C C_{s}$ has length $m$ and that sequence $C C_{t}$ has length $n$, we can obtain a two-dimensional

\footnotetext{
${ }^{\dagger}$ http://ictclas.org/
}

matrix with $C C_{s}$ as the row and $C C_{t}$ of $(m+1) \times(n+1)$ as the column.

\subsubsection{Needleman-Wunsch Algorithm}

The Needleman-Wunsch $(N W)$ algorithm is a typical global alignment and dynamic programming algorithm for comparing the similarity between the two sequences [30]. The optimal alignment path in matrix $M$ can be recursively solved based on the idea of dynamic programming.

In TongSACOM model, a sequence-alignment and improved TYCCL-based combined algorithm is proposed to evaluate the similarity between Chinese OOV.

Sequence Alignment Processing: Firstly, let the penalty factor of sequence alignment algorithm be $p=$ -0.05 and initialize Row $m+1$ and Column $n+1$ in the matrix. Secondly, evaluating all the pair of atomic concepts similarity based on the improved TYCCL similarity function $\operatorname{SIM}_{T}$ (Eq. (1)) and the remaining $m \times n$ elements value will be computed recursively in the matrix. The definition of the scoring function $f$ is shown in Eq. (3):

$$
\begin{aligned}
& f\left(A C_{s i}, A C_{t j}\right) \\
& =\left\{\begin{array}{l}
\operatorname{SIM}_{T}\left(A C_{s i}, A C_{t j}\right) \quad \text { if } A C_{s i} \neq="-" \text { and } A C_{t j} \neq \cdots-" \\
f\left(A C_{s i},-\right)=p=-0.05 \quad \text { if } A C_{t j}="-" \\
f\left(-, A C_{t j}\right)=p=-0.05 \quad \text { if } A C_{s i}="-"
\end{array}\right.
\end{aligned}
$$

Considering the feature of "back loading" that is prevalent in Chinese concepts [16], [28], we choose the end of two compound concepts, that is the element $M_{m n}$, as the starting point of the recursive algorithm in the scoring matrix. Please see Eq. (1) for the description of $\operatorname{SIM}_{T}$. The recursive rule is as described in Eq. (4). 


$$
M_{i j}=\max \left\{\begin{array}{l}
M_{(i+1)(j+1)}+f\left(A C_{s i}, A C_{t j}\right) \\
M_{(i)(j+1)}+p \\
M_{(i+1)(j)}+p
\end{array}\right.
$$

Thirdly, starting from $M_{m n}$ in the matrix, backtracking $M_{11}$ and it will be possible to obtain the optimal alignment path-contained matrix: $M_{(i)(j)}^{\prime}$, returned by Algorithm 1. It should be noted that, if there is more than one optimal alignment path, only one should be chosen. Here, a space character ("-") was inserted, as there were sequences of the two compound concepts for mapping that were equal in length, called $C C_{s i}$ and $C C_{t i}{ }^{\prime}$. The length of the two sequences is defined as $L$. The Algorithm 1 shows the details of getting the optimal alignment path processing between compound concepts based on the $N W$ algorithm.

Finally, we obtain Eq. (5) to compute the similarity between compound concepts based on alignment results and scoring function $f$ :

$$
\operatorname{SIM}_{N W}\left(C C_{s i}^{\prime}, C C_{t i}^{\prime}\right)=\sum_{i=1}^{|L|} \frac{f\left(A C_{s i}, A C_{t i}\right)}{|L|}
$$

\section{Experimental Results and Analysis}

This study used online Chinese encyclopedia knowledge bases as sources of the experimental data. In addition to the DBpedia (Chinese version) [23], we used a crawler toolkit, HTMLParser, to crawl and analyze the classification tree of Baidu Baike and Hudong. We did not only analyze the classification systems in Chinese open-source encyclopedia knowledge bases, but also extracted and analyzed the structured Infobox information included in all of the entry pages and organized them in the form of Chinese triples. All concepts for mapping are on the classification tree of Chinese online encyclopedias.

Eventually, the three large-scale ontologies for mapping are formed, as Shown in Table 2. The strategy for building ontology for Baidu Baike and Hudong Baike was based on the methods reported in Refs. [5], [31].

\subsection{Sequence Alignment Result Analysis}

For example, splitting a pair of compound concepts to be mapped in Baidu-DBpedia sub-mapping task: history, namely, “第二次工业革命 (the second industrial revolution)” and “第二次世界大战人物 (people of the second world war)," we can obtain two word sequences: [第二, 次, 工业革命] ([second, (measure word), industrial revolution]) and [第二, 次, 世界大战, 人物] ([second, (measure word), world war, people]). Based on the idea of dynamic programming, the two word-sequences are expressed in rows and columns which consist of the scoring matrix: $M_{(i)(j)}$.

Similarly using the example of combining the concepts: “第二次工业革命 (the second industrial revolution)” and “第二次世界大战人物 (people of the second world war)", matrix $M_{(i)(j)}^{\prime}$, which includes the optimal alignment path-which is resolved using Algorithm 1. The scoring matrix outputted by Algorithm 1 is shown in Fig. 4. The bold arrow indicates the optimal alignment path that is obtained. The bold diagonal arrow indicates that two atomic concepts at the end are mapped. The bold horizontal arrows indicate a space character "-" should be inserted in front of the atomic concept on the row where the sequence $C C_{s}$ is located. A bold vertical arrow indicates a space character "-" should be inserted in front of the atomic concept on the column where the sequence $C C_{t}$ is located.

The optimal alignment results of sequence [第二, 次, 工业革命] and [第二, 次, 世界大战, 人物] are shown in Fig. 5.

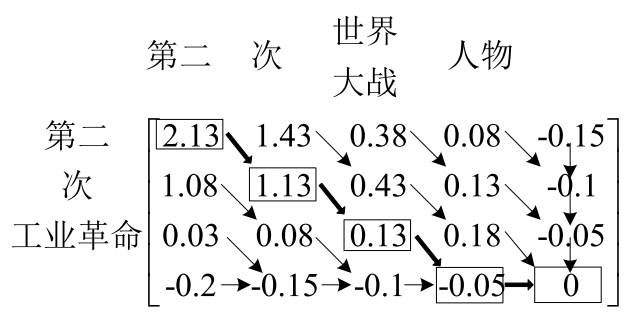

Fig. 4 The scoring matrix for Example 2.

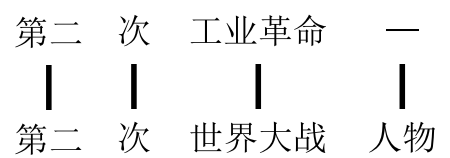

Fig. 5 The correct sequence alignment result for Example 2.

Table 2 Information on chinese encyclopedia knowledge bases

\begin{tabular}{ccccc}
\hline & Items & Baidu Baike & Hudong & DBpedia \\
\hline \multirow{3}{*}{ Concept } & Top Categories & 13 & 13 & 23 \\
\cline { 2 - 5 } & Number of Concepts & 1,336 & 29,276 & 106,023 \\
\cline { 2 - 5 } & Chinese Literal Triples & 1,323 & 29,263 & 106,000 \\
\hline \multirow{3}{*}{ Infobox } & Number of Infoboxes & 214,732 & 257,215 & 204,822 \\
\cline { 2 - 5 } & Number of Properties & 21,152 & 1,061 & 18,206 \\
\cline { 2 - 5 } & Chinese Literal Triples & $1,698,149$ & $2,161,616$ & $4,077,898$ \\
\hline \multirow{2}{*}{ Individual } & Infobox Percentage & $2.30 \%$ & $10.10 \%$ & $19.74 \%$ \\
\cline { 2 - 5 } & Chinese Literal Triples & $9,346,184$ & $2,545,447$ & $1,037,557$ \\
\hline
\end{tabular}




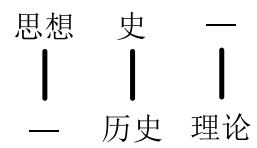

Fig. 6 The correct sequence alignment result for Example 1.

$$
\begin{aligned}
& \text { 历史 理论 }
\end{aligned}
$$

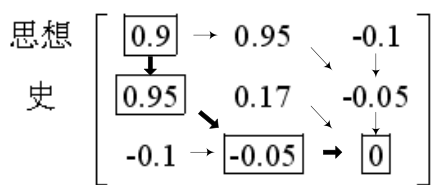

Fig. 7 The scoring matrix for Example 1.

Here, we reexamined the two examples of computing similarity that were mentioned before.

Example 1: $C C_{s}=$ [思想, 史] ([thought, history]), $C C_{t}=$ [历史, 理论] ([history, theory]).

The similarity value of the component concept that is obtained using Eq. (2) is:

$$
\operatorname{Sim}\left(C C_{s}, C C_{t}\right)=(1.0+1.0) / 2=1.0
$$

Moreover, the alignment effect on the two compound concept sequences that returned by the sequence alignment algorithm is shown in Fig. 6 .

The corresponding scoring matrix is shown in Fig. 7.

The final similarity between the compound concepts should be:

$$
\operatorname{SIM}_{N W}\left(C C_{s i}, C C_{t i}^{\prime}\right)=(-0.05+1.0-0.05) / 3=0.3
$$

Example 2: $C C_{s}=$ [第二, 次, 工业革命] ([second, (measure word), industrial revolution]), $C C_{t}=$ [第二, 次, 世界大战, 人物] ([second, (measure word), world war, people]).

The component concept similarity value that is obtained using Eq. (2) is $\operatorname{Sim}\left(C C_{s}, C C_{t}\right)=1.0$. This is because atomic concept “次” has a phenomenon of "polysemy”. Specifically, the lexical unit “次” has multiple items of TYCCL (extended version) code, among which the code "Dn04B03=" determines that two atomic lexical units, "第 二” and “次”, are equivalent. Thus, according to Eq. (2) given by Refs. [14], there are four cases in which the mapping result of the atomic concept is 1.0 : <第二, 次 $>=1.0$; $<$ 第二, 第二 $>=1.0 ;<$ 次, 第二 $>=1.0 ;$ and $<$ 次, 次 $>=$ 1.0. Put these in Eq. (2), and

$$
\operatorname{Sim}\left(C C_{s}, C C_{t}\right)=(1.0+1.0+1.0+1.0) / 4=1.0
$$

The final compound concept similarity value, which is computed based on Algorithm 1, should be:

$$
\begin{aligned}
\operatorname{SIM}_{N W}\left(C C_{s i}^{\prime}, C C_{t i}^{\prime}\right) & =(1.0+1.0+0.18-0.05) / 4 \\
& =0.5325
\end{aligned}
$$

Its sequence matching results are shown in Fig. 5.

It can be seen that there are no equivalent relationship between the two compound concepts in Example 1
Table 3 Statistics on standard reference mapping pairs of Baidu Hudong mapping task

\begin{tabular}{ccccc}
\hline \multirow{2}{*}{$\begin{array}{c}\text { Mapping } \\
\text { Task }\end{array}$} & $\begin{array}{c}\text { Top } \\
\text { Category }\end{array}$ & $\begin{array}{c}\text { Number of } \\
\text { Concepts in } \\
\text { Baidu-Baike }\end{array}$ & $\begin{array}{c}\text { Number of } \\
\text { Concepts in } \\
\text { Hudong }\end{array}$ & $\begin{array}{c}\text { Reference } \\
\text { Mapping } \\
\text { Pairs }\end{array}$ \\
\hline \multirow{7}{*}{ Baidu- } & Person & 120 & 1497 & 57 \\
\cline { 2 - 5 } Hudong & Science & 157 & 2323 & 62 \\
\cline { 2 - 5 } & Society & 102 & 3937 & 60 \\
\cline { 2 - 5 } & Artory & 118 & 2093 & 54 \\
\cline { 2 - 5 } & Nature & 104 & 1506 & 55 \\
\cline { 2 - 5 } & Sports & 165 & 5688 & 71 \\
\cline { 2 - 5 } & Geography & 133 & 258 & 59 \\
\hline
\end{tabular}

and Example 2 at all. The wrong conclusion is given by the traditional method that their similarity values are 1.0, which is extremely high. Conversely, the similarity values that are obtained using Algorithm 1 are more reasonable. Thus, when the features of Chinese, such as word ordersensitive and polysemy are considered, using the global alignment algorithm based on the Needleman-Wunsch algorithm can effectively avoid the incorrect mapping that the traditional method represented by Eq. (2) may cause. Meanwhile, when mapping compound concepts, if the semantic orders of atomic concepts in word sequences are essentially the same, then Algorithm 1 is consistent with the traditional method.

In summary, the method for computing similarity in element-level concepts based on global sequence alignment is more advantageous and reasonable than the traditional method for open-domain and large-scale Chinese ontology mapping tasks.

\subsection{Evaluation Indicators}

In this study, the Precision, Recall, and F-measure (F1) were used as the final evaluation criteria.

Precision $(\mathrm{P})=$ Correct mapping pairs / total output mapping pairs $\times 100 \%$

Recall $(\mathrm{R})=$ Correct mapping pairs $/$ total reference mapping pairs $\times 100 \%$

F-measure $(\mathrm{F} 1)=2 \times \mathrm{P} \times \mathrm{R} /(\mathrm{P}+\mathrm{R}) \times 100 \%$

We invite four Chinese senior grade students from Capital University of Economics and Business to use the manual identification and manual annotation-based method for annotating the equivalence relationships between concepts existing in the top classification tree of DBpedia, BaiduBaike and Hudong. The annotation results are treated as the reference mapping pairs for the Chinese ontology mapping task. The number of reference mapping pairs in the three mapping tasks is shown in Tables 3, 4 and 5:

\subsection{Evaluation of the Chinese Ontology Mapping Results}

This study used four different algorithms to evaluate the 
results of large-scale Chinese ontology mapping in LOD environment. The first one is the Edit-Distance based algorithm, which can be commonly used in different languages [32]. The second one is the traditional TYCCLbased algorithm (TYCCL algorithm) that computes the similarity between Chinese atomic concepts [17]. The third one is the ELOMC system proposed by Li et al. [14]. The fourth one is the TongSACOM proposed in this study.

To ensure fairness, the similarity threshold value will be set to $t=0.9$, which is used to determine the equivalent relationships between concepts in each algorithm. The evaluation results for the three mapping tasks are shown in Tables 6,7 and 8 .

A conclusion can be get from Baidu-Hudong mapping

Table 4 Statistics on standard reference mapping pairs of Hudong DBpedia mapping task

\begin{tabular}{ccccc}
\hline \multirow{2}{*}{$\begin{array}{c}\text { Mapping } \\
\text { Task }\end{array}$} & $\begin{array}{c}\text { Top } \\
\text { Category }\end{array}$ & $\begin{array}{c}\text { Number of } \\
\text { Concepts in } \\
\text { Hudong }\end{array}$ & $\begin{array}{c}\text { Number of } \\
\text { Concepts in } \\
\text { DBpedia }\end{array}$ & $\begin{array}{c}\text { Reference } \\
\text { Mapping } \\
\text { Pairs }\end{array}$ \\
\hline \multirow{2}{*}{$\begin{array}{c}\text { Hudong- } \\
\text { DBpedia }\end{array}$} & Person & 1497 & 4737 & 380 \\
\cline { 2 - 5 } & Science & 2323 & 156 & 33 \\
\cline { 2 - 5 } & Society & 3937 & 10676 & 303 \\
\cline { 2 - 5 } & History & 2093 & 5648 & 524 \\
\hline
\end{tabular}

Table 5 Statistics on standard reference mapping pairs of Baidu DBpedia mapping task

\begin{tabular}{ccccc}
\hline \multirow{2}{*}{$\begin{array}{c}\text { Mapping } \\
\text { Task }\end{array}$} & $\begin{array}{c}\text { Top } \\
\text { Category }\end{array}$ & $\begin{array}{c}\text { Number of } \\
\text { Concepts in } \\
\text { Baidu-Baike }\end{array}$ & $\begin{array}{c}\text { Number of } \\
\text { Concepts in } \\
\text { DBpedia }\end{array}$ & $\begin{array}{c}\text { Reference } \\
\text { Mapping } \\
\text { Pairs }\end{array}$ \\
\hline & Person & 120 & 4737 & 26 \\
\cline { 2 - 5 } Baidu- & Society & 102 & 10676 & 28 \\
\cline { 2 - 5 } DBpedia & History & 118 & 5648 & 11 \\
\cline { 2 - 5 } & Art & 84 & 1908 & 25 \\
\cline { 2 - 5 } & Geography & 133 & 37936 & 95 \\
\hline
\end{tabular}

result as shown in Table 6. The Precision of TongSACOM was, on average, approx. $38 \%$ and $36 \%$ higher than that for TYCCL algorithm and ELOMC, respectively. Its Recall was, on average, approx. $14 \%$ and $3 \%$ higher than the Edit Distance and TYCCL algorithm but is roughly equal to ELOMC. Its average overall performance (F-measure) values were 7\%, 22\% and 20\% higher than the Edit-Distance, TYCCL algorithm and the ELOMC system respectively.

Hudong-DBpedia mapping result presented in Table 7 shows that the Precision of TongSACOM was, on average, approx. $1 \%, 10 \%$ and $11 \%$ higher than that for EditDistance, TYCCL algorithm and ELOMC, respectively. Its Recall was, on average, approx. $6 \%$ and $1 \%$ higher than that for Edit-Distance and TYCCL algorithm respectively, and is essentially equal to ELOMC. The average F1 values of TongSACOM were 3\%, 6\% and 6\% higher than the other three algorithms respectively.

Table 8 has shown us that, while facing the BaiduDBpedia mapping task, the Precision of TongSACOM is, on average, approx. $39 \%$ and $43 \%$ higher than that for TYCCL algorithm and ELOMC. Its Recall was, on average, approx. $17 \%, 6 \%$ and $3 \%$ higher than that for Edit-Distance, TYCCL algorithm and ELOMC respectively. The average F1 values were $8 \%, 26 \%$ and $30 \%$ higher than the other three algorithms respectively.

In addition, under the conditions of 4 levels of Algorithm factor (Edit-Distance, TYCCL algorithm, ELOMC and TongSACOM) and 72 samples, this study assumes that samples are independent of each other and follow normal distribution for conducting a Significance Test (OneWay ANOVA). We observe that, under 0.05 of the significance level, Precision of the four algorithms is significantly different affected by the Between Groups $\left(F_{0.05}(3,68)<\right.$ 27.496, $p=0.000<0.05$ ); Recall of the four algorithms is significantly different affected by the Between Groups $\left(F_{0.05}(3,68)<5.194, \quad p=0.003<0.05\right)$; and the overall performance (F-measure) of the four algorithms is also significantly different affected by the Between Groups $\left(F_{0.05}(3,68)<9.474, p=0.000<0.05\right)$.

Table 6 Results and evaluation of four typical similarity algorithms of Baidu - Hudong mapping task

\begin{tabular}{|c|c|c|c|c|c|c|c|c|c|c|c|c|c|}
\hline \multirow{2}{*}{$\begin{array}{l}\text { Mapping } \\
\text { Task }\end{array}$} & \multirow{2}{*}{$\begin{array}{c}\text { Top } \\
\text { Category }\end{array}$} & \multicolumn{3}{|c|}{$\begin{array}{l}\text { Edit-Distance } \\
\text { Algorithm[32] }\end{array}$} & \multicolumn{3}{|c|}{$\begin{array}{c}\text { Original TYCCL-Based } \\
\text { Algorithm[17] }\end{array}$} & \multicolumn{3}{|c|}{ ELOMC[14] } & \multicolumn{3}{|c|}{ TongSACOM } \\
\hline & & $\mathrm{P}$ & $\mathrm{R}$ & F1 & $\mathrm{P}$ & $\mathrm{R}$ & $\mathrm{F} 1$ & $\mathrm{P}$ & $\mathrm{R}$ & F1 & $\mathrm{P}$ & $\mathrm{R}$ & $\mathrm{F} 1$ \\
\hline \multirow{9}{*}{$\begin{array}{l}\text { Baidu- } \\
\text { Hudong }\end{array}$} & Person & 1.000 & 0.526 & 0.690 & 0.657 & 0.772 & 0.710 & 0.943 & 0.877 & 0.909 & 0.980 & 0.877 & 0.926 \\
\hline & Science & 1.000 & 0.742 & 0.852 & 0.422 & 0.790 & 0.551 & 0.387 & 0.774 & 0.516 & 0.982 & 0.774 & 0.866 \\
\hline & Society & 1.000 & 0.567 & 0.723 & 0.640 & 0.800 & 0.711 & 0.622 & 0.850 & 0.718 & 0.662 & 0.817 & 0.731 \\
\hline & History & 1.000 & 0.611 & 0.759 & 0.607 & 0.630 & 0.618 & 0.607 & 0.685 & 0.643 & 1.000 & 0.685 & 0.813 \\
\hline & Art & 1.000 & 0.727 & 0.842 & 0.769 & 0.909 & 0.833 & 0.729 & 0.927 & 0.816 & 1.000 & 0.927 & 0.962 \\
\hline & Nature & 1.000 & 0.704 & 0.826 & 0.369 & 0.775 & 0.500 & 0.364 & 0.775 & 0.495 & 0.965 & 0.775 & 0.859 \\
\hline & Sports & 1.000 & 0.763 & 0.865 & 0.554 & 0.780 & 0.648 & 0.516 & 0.814 & 0.632 & 0.980 & 0.814 & 0.889 \\
\hline & Geography & 1.000 & 0.691 & 0.817 & 0.470 & 0.804 & 0.593 & 0.491 & 0.845 & 0.621 & 0.988 & 0.835 & 0.905 \\
\hline & Average & 1.000 & 0.666 & 0.797 & 0.561 & 0.782 & 0.645 & 0.582 & 0.818 & 0.669 & 0.945 & 0.813 & 0.869 \\
\hline
\end{tabular}


Table 7 Results and evaluation of four typical similarity algorithms of Hudong - DBpedia mapping task

\begin{tabular}{|c|c|c|c|c|c|c|c|c|c|c|c|c|c|}
\hline \multirow{2}{*}{$\begin{array}{l}\text { Mapping } \\
\text { Task }\end{array}$} & \multirow{2}{*}{$\begin{array}{c}\text { Top } \\
\text { Category }\end{array}$} & \multicolumn{3}{|c|}{$\begin{array}{l}\text { Edit-Distance } \\
\text { Algorithm[32] }\end{array}$} & \multicolumn{3}{|c|}{$\begin{array}{c}\text { Original TYCCL-Based } \\
\text { Algorithm[17] }\end{array}$} & \multicolumn{3}{|c|}{ ELOMC[14] } & \multicolumn{3}{|c|}{ TongSACOM } \\
\hline & & $\mathrm{P}$ & $\mathrm{R}$ & $\mathrm{F} 1$ & $\mathrm{P}$ & $\mathrm{R}$ & F1 & $\mathrm{P}$ & $\mathrm{R}$ & $\mathrm{F} 1$ & $\mathrm{P}$ & $\mathrm{R}$ & $\mathrm{F} 1$ \\
\hline & Person & 0.973 & 0.861 & 0.913 & 0.955 & 0.889 & 0.921 & 0.949 & 0.924 & 0.936 & 0.956 & 0.924 & 0.940 \\
\hline & Science & 0.939 & 0.939 & 0.939 & 0.838 & 0.939 & 0.886 & 0.886 & 0.939 & 0.912 & 1.000 & 0.939 & 0.969 \\
\hline Hudong- & Society & 0.964 & 0.894 & 0.928 & 0.793 & 0.983 & 0.878 & 0.794 & 0.993 & 0.883 & 0.990 & 0.993 & 0.992 \\
\hline \multirow[t]{3}{*}{ DBpedia } & History & 0.987 & 0.987 & 0.987 & 0.992 & 0.989 & 0.990 & 0.979 & 0.992 & 0.986 & 0.994 & 0.992 & 0.993 \\
\hline & Art & 0.984 & 0.938 & 0.960 & 0.823 & 0.990 & 0.899 & 0.740 & 0.995 & 0.849 & 0.989 & 1.000 & 0.994 \\
\hline & Average & 0.969 & 0.924 & 0.946 & 0.880 & 0.958 & 0.915 & 0.870 & 0.969 & 0.913 & 0.986 & 0.970 & 0.978 \\
\hline
\end{tabular}

Table 8 Results and evaluation of four typical similarity algorithms of Baidu - DBpedia mapping task

\begin{tabular}{|c|c|c|c|c|c|c|c|c|c|c|c|c|c|}
\hline \multirow{2}{*}{$\begin{array}{l}\text { Mapping } \\
\text { Task }\end{array}$} & \multirow{2}{*}{$\begin{array}{c}\text { Top } \\
\text { Category }\end{array}$} & \multicolumn{3}{|c|}{$\begin{array}{l}\text { Edit-Distance } \\
\text { Algorithm[32] }\end{array}$} & \multicolumn{3}{|c|}{$\begin{array}{c}\text { Original TYCCL-Based } \\
\text { Algorithm[17] }\end{array}$} & \multicolumn{3}{|c|}{ ELOMC[14] } & \multicolumn{3}{|c|}{ TongSACOM } \\
\hline & & $\mathrm{P}$ & $\mathrm{R}$ & $\mathrm{F} 1$ & $\mathrm{P}$ & $\mathrm{R}$ & $\mathrm{F} 1$ & $\mathrm{P}$ & $\mathrm{R}$ & $\mathrm{F} 1$ & $\mathrm{P}$ & $\mathrm{R}$ & $\mathrm{F} 1$ \\
\hline & Person & 1.000 & 0.692 & 0.818 & 0.452 & 0.731 & 0.559 & 0.733 & 0.846 & 0.786 & 0.963 & 1.000 & 0.981 \\
\hline & Society & 1.000 & 0.679 & 0.809 & 0.639 & 0.821 & 0.719 & 0.500 & 0.821 & 0.622 & 0.955 & 0.750 & 0.840 \\
\hline Baidu- & History & 1.000 & 0.727 & 0.842 & 0.692 & 0.818 & 0.750 & 0.188 & 0.818 & 0.305 & 0.900 & 0.818 & 0.857 \\
\hline \multirow[t]{3}{*}{ DBpedia } & Art & 1.000 & 0.760 & 0.864 & 0.639 & 0.920 & 0.754 & 0.821 & 0.920 & 0.868 & 1.000 & 1.000 & 1.000 \\
\hline & Geography & 1.000 & 0.853 & 0.920 & 0.421 & 0.979 & 0.589 & 0.413 & 1.000 & 0.585 & 0.989 & 0.989 & 0.989 \\
\hline & Average & 1.000 & 0.742 & 0.851 & 0.569 & 0.854 & 0.674 & 0.531 & 0.881 & 0.633 & 0.961 & 0.912 & 0.934 \\
\hline
\end{tabular}

The Precision of TongSACOM is slightly lower than the Edit-Distance algorithm in Baidu-Hudong and BaiduDBpedia mapping task. It is mainly because a few concepts are controversial or improper classified as a pair of synonym in TYCCL, such as: “作家 (writer)” and “女作家 (female writer)”, “文学家 (litterateurs)” and “散文家 (essayist)", “民族 (nation)” and “中华民族 (the Chinese nation)", “刑法 (Criminal laws)” and “刑事 (criminal)”, “辛亥革命 (the Revolution of 1911 (the Chinese bourgeois democratic revolution led by Dr, Sun Yat-sen which overthrew the Qing Dynasty))" and “革命 (revolution)" etc. If they appear in the final result, we consider them as the wrong mapping pairs. This situation appears more in the Society sub-mapping task of Baidu-Hudong but rarely appears in others; furthermore, the average Precision of TongSACOM in the three groups of Chinese Encyclopedia mapping tasks are 94.5\%, 98.6\% and $96.1 \%$ respectively, which are still completely within the acceptable range. While the Edit-Distance algorithm can only mechanically compare the concept similarity on the literal similarity level, which totally ignores the semantic similarity between concepts and will inevitably lead to its recall in all mapping tasks were significantly lower than those of the other systems. By comparison, TongSACOM firstly introduces the TYCCL (extended version) as the semantic dictionary and then improves the original TYCCL algorithm in order to adapt it to the large-scale Chinese ontology mapping task; therefore, the Recall of TongSACOM is significantly higher than Edit-Distance algorithm, which

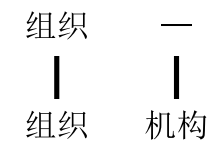

Fig. 8 The example 1 of unreasonable sequence alignment result

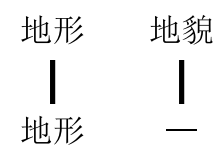

Fig.9 The example 2 of unreasonable sequence alignment result

eventually makes the overall average performance (Fmeasure) of TongSACOM is higher than Edit-Distance algorithm in all the three groups of Chinese Encyclopedias mapping results.

The specific Recall of Society and Geography in Baidu-Hudong mapping task is slightly lower than the ELOMC. The reason is that there were thought to be correct synonym mapping pairs such as "组织 (organization)" and “组织机构 (organizational structure)”, “地形地貌 (topography)" and “地形 (topography)" in the Society and Geography mapping results, but due to the length of the two sequences to be mapped is 1 and 2 respectively, a differential of 2 , which ultimately led to the wrong sequence alignment result. As shown in Figs. 8 and 9. Therefore, while the length of two sequences to be mapped corresponding to the 
correct synonym mapping pairs is various, sequence alignment algorithm is easy to cause the unreasonable similarity value. This situation rarely appears in the three groups of Chinese Encyclopedia mapping tasks. Compared to the previous algorithm that completely ignored the rules of wordorder sensitivity and polysemy in Chinese, TongSACOM has an obvious advantage in the promotion of Precision, which eventually makes the overall average performance (Fmeasure) of TongSACOM is obviously higher than the traditional TYCCL algorithm and the ELOMC system in all the three groups of Chinese Encyclopedias mapping results.

In summary, the overall performance (F-measure) of TongSACOM is the best of its kind. Its Precision is obviously higher than traditional TYCCL algorithm and ELOMC. The Recall of the TongSACOM model is higher than Edit-Distance and traditional TYCCL algorithm, and is essentially equal to ELOMC system.

\section{Conclusion}

Linked Open Data at Schema-Level and knowledge described in Chinese is an important part of the LOD project. Previous work generally ignored the rules of word-order sensitivity and polysemy in Chinese or could not deal with the out-of-vocabulary mapping task. In this paper, the further and systematic research is done at the aspect of how to build Chinese linked open data at Schema-Level, and a novel Chinese ontology mapping prototype (TongSACOM) in LOD scenario is proposed.

Based on the TYCCL and global sequence alignment theory, firstly, for a pair of atomic Chinese concepts, an improved TYCCL-based algorithm is proposed to compute the similarity between two atomic concepts; secondly, for a pair of compound Chinese concepts, based on the semantic features of Chinese, a novel strategy for Chinese ontology alignment is proposed by incorporating sequence alignment from bioinformatics.

This study may have important practical significance for enriching the schema-interlinkings between the Chinese Knowledge Bases in LOD environment and can hasten some new online applications in different areas.

\section{Acknowledgments}

This work was supported by BPSSP (15ZHB011), JSPS KAKENHI Grant (No.15K00332) and Capital University of Economics and Business Research Grant.

\section{References}

[1] T. Berners-Lee, J. Hendler, and O. Lassila, "The semantic web," Scientific American, vol.284, no.5, pp.28-37, 2001.

[2] W.N. Borst, "Construction of engineering ontologies for knowledge sharing and reuse," Universiteit Twente, 1997.

[3] C. Bizer, T. Heath, K. Idehen, and T. Berners-Lee, "Linked data on the web," Proc. 17th International Conference on World Wide Web, pp.1265-1266, Beijing, China, April 2008.

[4] X. Niu, X. Sun, H. Wang, S. Rong, G. Qi, and Y. Yu, "Zhishi.meweaving chinese linking open data," Proc. International Semantic
Web Conference, Bonn, Germany, pp.205-220, Oct. 2011.

[5] Z.-C. Wang, Z.-G. Wang, J.-Z. Li, and J.Z. Pan, "Knowledge extraction from Chinese wiki encyclopedias," Journal of Zhejiang University SCIENCE C, vol.13, no.4, pp.268-280, 2012.

[6] P. Jain, P. Hitzler, A.P. Sheth, K. Verma, and P.Z. Yeh, "Ontology alignment for linked open data," Proc. International Semantic Web Conference, Shanghai, China, pp.402-417, Nov. 2010.

[7] S. Melnik, H. Garcia-Molina, and E. Rahm, "Similarity flooding: A versatile graph matching algorithm and its application to schema matching," Proc. IEEE 18th International Conference on Data Engineering, San Jose, CA, pp.117-128, 2002.

[8] W. Cohen, P. Ravikumar, and S. Fienberg, "A comparison of string metrics for matching names and records," Proc. Kdd workshop on data cleaning and object consolidation, vol.3, pp.73-78, Aug. 2003.

[9] F. Guinchiglia and M. Yatskevich, "Element level semantic matching," Meaning Coordination and Negotiation, University of Trento, Italy, June 2004.

[10] M.M. Stark and R.F. Riesenfeld, "Wordnet: An electronic lexical database," Proc. 11th Eurographics Workshop on Rendering, vol.37, 1998.

[11] A. Isaac, L. Van Der Meij, S. Schlobach, and S.H. Wang, "An empirical study of instance-based ontology matching," Proc. 6th International Semantic Web Conference, 2nd Asian Semantic Web Conference, Busan, Korea, pp.253-266, 2007.

[12] A. Nikolov, V. Uren, E. Motta, and A.D. Roeck, "Integration of semantically annotated data by the KnoFuss architecture," Proc. International Conference on Knowledge Engineering and Knowledge Management, Acitrezza, Italy, pp.265-274, Sept. 2008.

[13] Q. Zhong, H.Y. Li, J.Z. Li, G.T. Xie, J. Tang, and Y. Pan, "A gauss function based approach for unbalanced ontology matching," Proc. ACM SIGMOD, Rhode Island, USA, pp.669-680, 2009.

[14] J. Li, M. Zhu, C. Liu, and Z.Q. Yang, "Research and implementation on Chinese ontology mapping," J. Chinese Information Processing, vol.21, no.4, pp.27-33, 2007.

[15] Z.D. Dong, Q. Dong, and C.L. Hao, "Theoretical findings of HowNet," Journal of Chinese Information Processing, vol.21, no.4, pp.3-9, 2007.

[16] B.F. Lu, "Word order dominance and its cognitive explanation," Contemporary Linguistics, vol.7, no.1, pp.1-15, 2005.

[17] J.L. Tian and W. Zhao, "Words similarity algorithm based on TongyiciCilin in semantic web adaptive learning system," J. Jilin University, vol.28, no.6, pp.602-608, 2010.

[18] J. Volz, C. Bizer, M. Gaedke, and G. Kobilarov, "Silk-A Link Discovery Framework for the Web of Data," Proc. Workshop on Linked Data on the Web, Madrid, Spain, 2009.

[19] J. Volz, C. Bizer, M. Gaedke, and G. Kobilarov, "Discovering and maintaining links on the web of data," Proc. International Semantic Web Conference, Chantilly, VA, USA, pp.650-665, 2009.

[20] O. Hassanzadeh, L. Lim, A. Kementsietsidis, and M. Wang, "A Declarative Framework for Semantic Link Discovery over Relational Data," Proc. 18th International Conference on World Wide Web, Madrid, Spain, pp.1101-1102, April 2009.

[21] C. Bizer and A. Seaborne, "D2RQ-treating non-RDF databases as virtual RDF graphs," Proc. International Semantic Web Conference, 2005.

[22] Z.C. Wang, J.Z. Li, and J. Tang, "Boosting cross-lingual knowledge linking via concept annotation," Proc. Twenty-Third International Joint Conference on Artificial Intelligence, Beijing, China, pp.27332739, 2013.

[23] C. Bizer, J. Lehmann, G. Kobilarov, S. Auer, C. Becker, R. Cyganiak, and S. Hellmann, "DBpedia-A crystallization point for the web of data," J. Web Semantics, vol.7, no.3, pp.154-165, 2009.

[24] J.J. Mei and Y.M. Zhu, TongyiciCilin, Shanghai Lexicographical Publishing House, Shanghai, China, 1983.

[25] C.Z. Zhang, "Research on Synonyms Dictionary-Based on Recognition of Synonyms," J. Huaiyin Institute of Technology, vol.13, no.1, pp.59-62, 2004. 
[26] T. Xia, "Study on Chinese words semantic similarity computation," J. Computer Engineering, vol.33, no.6, pp.191-194, 2007.

[27] W.R. Wang, "Dynamic construction of the lexical knowledge system: Approaches and tools implementation," Institute of Scientific and Technical Information of China, pp.58-75, 2008.

[28] S.Q. Cheng, A Study on the Priority Sequence of Multiple-attribute Phrases in Modern Chinese, Doctoral Dissertation of Central China Normal University, 2009.

[29] J.C. Setubal and J. Meidanis, Introduction to computational molecular biology, PWS Publishing, Boston, MA, 1997.

[30] S.B. Needleman and C.D. Wunsch, "A general method applicable to the search for similarities in the amino acid sequence of two proteins," Journal of Molecular Biology, vol.48, no.3, pp.443-453, 1970.

[31] T. Wang, J. Song, R.H. Di, and Y. Liang, "A thesaurus and online encyclopedia merging method for large scale domain-ontology automatic construction," Proc. 6th International Conference on Knowledge Science, Engineering and Management, Dalian, China, pp.132-146, Aug. 2013.

[32] V. Levenshtein, "Binary codes capable of correcting deletions, insertions, and reversals," Soviet Physics Doklady, vol.10, no.8, pp.707$710,1966$.

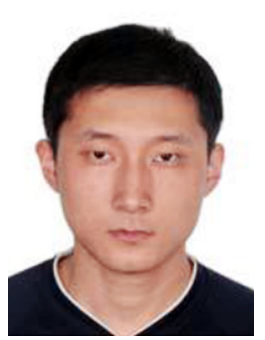

Ting Wang received his B.S. and Ph.D. degree in Computer Science and Technology from the Beijing University of Technology in 2008 and 2014, respectively. In 2012, he was a research student at Institute of Automation, Chinese Academy of Sciences, Beijing, China. $\mathrm{He}$ is currently a lecturer at the Information School, Capital University of Economics and Business. His research interests include semantic web, knowledge discovery, and natural language processing.

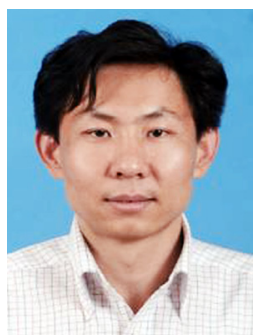

Tiansheng Xu received his B.E. (Civil Engineering) degree from Beijing Institute of $\mathrm{Ar}$ chitecture in 1995, M.E. degree and D.E. degree from University of Toyama, Toyama, Japan in 1999 and 2002, respectively. He is currently a professor at the Information School, Capital University of Economics and Business. His research interests include natural language processing, cognitive science, and linguistics.

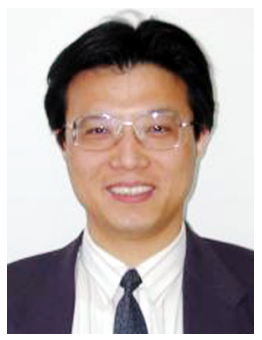

Zheng Tang received the B.S. degree from Zhejiang University, Zhejiang, China in 1982 and an M.S. degree and a D.E. degree from Tsinghua University, Beijing, China in 1984 and 1988, respectively. From 1988 to 1989, he was an instructor in the institute of microelectronics at Tsinghua University. From 1990 to 1999, he was an associate professor in the department of electrical and electronic engineering, Miyazaki University, Miyazaki, Japan. In 2000, he joined University of Toyama, Toyama, Japan as a professor of the department of intellectual information systems. His current research interests include intellectual information technology, neural networks, and optimizations.

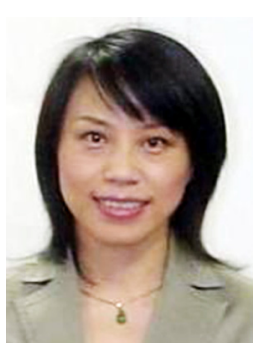

Yuki Todo received the B.S. degree from Zhejiang University, Zhejiang, China, the M.S. degree from Beijing University of Posts and Telecommunications, Beijing, China, and the D.E. degree from Kanazawa University, Kanazawa, Japan, in 1983, 1986, and 2005, respectively. From 1987 to 1989 , she was an Assistant Professor with the Institute of Microelectronics, Shanghai Jiaotong University, Shanghai, China. From 1989 to 1990, she was a research student at Nagoya University, Nagoya, Japan. From 1990 to 2000, she was a Senior Engineer with Sanwa Newtech Inc., Miyazaki, Japan. From 2000 to 2011, she worked with Tateyama Systems Institute, Toyama, Japan. In 2012, she joined Kanazawa University, where she is now an Associate Professor with the Faculty of Electrical and Computer Engineering. Her current research interests include multiplevalued logic, neural networks, and optimization. 